\title{
Loss of YABBY2-Like Gene Expression May Underlie the Evolution of the Laminar Style in Canna and Contribute to Floral Morphological Diversity in the Zingiberales
}

\author{
Kelsie Morioka ${ }^{1}$, Roxana Yockteng ${ }^{1,2,3}$, Ana M. R. Almeida ${ }^{1,4}$ and Chelsea D. Specht ${ }^{1 *}$ \\ ${ }^{1}$ Department of Plant and Microbial Biology, Department of Integrative Biology and the University and Jepson Herbaria, \\ University of California at Berkeley, Berkeley, CA, USA, ${ }^{2}$ Corporación Colombiana de Investigación Agropecuaria \\ (CORPOICA), Centro de Investigaciones Tibaitatá, Tibaitatá, Colombia, ${ }^{3}$ Institut de Systématique, Évolution, Biodiversité, \\ UMR 7205 Centre National de la Recherche Scientifique, Muséum National d'Histoire Naturelle, Paris, France, ${ }^{4}$ Programa de \\ Pós-graduação em Genética e Biodiversidade, Universidade Federal da Bahia, Salvador, Brazil
}

OPEN ACCESS

Edited by:

Jocelyn Hall,

University of Alberta, Canada

Reviewed by:

Bruce Veit,

AgResearch, New Zealand Marcelo Carnier Dornelas, Universidade Estadual de Campinas,

Brazil

*Correspondence:

Chelsea D. Specht cdspecht@berkeley.edu

Specialty section:

This article was submitted to Plant Evolution and Development,

a section of the journal

Frontiers in Plant Science

Received: 15 August 2015 Accepted: 22 November 2015 Published: 16 December 2015

Citation:

Morioka K, Yockteng R, Almeida AMR and Specht CD (2015) Loss of YABBY2-Like Gene Expression May Underlie the Evolution of the Laminar

Style in Canna and Contribute to Floral Morphological Diversity in the Zingiberales. Front. Plant Sci. 6:1106. doi: 10.3389/fpls.2015.01106
The Zingiberales is an order of tropical monocots that exhibits diverse floral morphologies. The evolution of petaloid, laminar stamens, staminodes, and styles contributes to this diversity. The laminar style is a derived trait in the family Cannaceae and plays an important role in pollination as its surface is used for secondary pollen presentation. Previous work in the Zingiberales has implicated YABBY2-like genes, which function in promoting laminar outgrowth, in the evolution of stamen morphology. Here, we investigate the evolution and expression of Zingiberales YABBY2-like genes in order to understand the evolution of the laminar style in Canna. Phylogenetic analyses show that multiple duplication events have occurred in this gene lineage prior to the diversification of the Zingiberales. Reverse transcription-PCR in Canna, Costus, and Musa reveals differential expression across floral organs, taxa, and gene copies, and a role for YABBY2-like genes in the evolution of the laminar style is proposed. Selection tests indicate that almost all sites in conserved domains are under purifying selection, consistent with their functional relevance, and a motif unique to monocot YABBY2-like genes is identified. These results contribute to our understanding of the molecular mechanisms underlying the evolution of floral morphologies.

Keywords: YABBY, YABBY2, gene evolution, gene expression, floral development, plant evolution, Zingiberales, Canna

\section{INTRODUCTION}

Laminar outgrowth is a key process in the development of lateral organs, facilitating light capture and gas exchange (leaves), pollinator attraction (petals and sometimes other floral organs), and protection of the floral bud (sepals and bracts). It has been hypothesized that, at the molecular level, laminar outgrowth is promoted by the juxtaposition of abaxial and adaxial cell fates 
(Waites and Hudson, 1995). Numerous studies have identified and characterized the genes involved in establishing abaxialadaxial polarity and the promotion of laminar expansion of lateral organs. In Arabidopsis, studies of loss-of-function and gain-of-function mutants indicate that KANADI, ARF3/ETT, and $A R F 4$ genes play roles in specifying abaxial cell fate, while HD-ZIPIII (PHABULOSA, PHAVOLUTA, and REVOLUTA) and $A S 1 / A S 2$ genes play roles in specifying adaxial cell fate (for more detailed review of these studies, see Bowman et al., 2002; Yamaguchi et al., 2012). YABBY genes are likely to act downstream in the adaxial-abaxial polarity regulatory network, promoting blade outgrowth at the abaxial-adaxial boundary (Husbands et al., 2009).

The $Y A B B Y$ gene family was believed to be specific to seed plants, but $Y A B B Y$ genes have been found in the green alga Micromonas pusilla (Worden et al., 2009). Since these genes have not been found in any other non-seed plants, it is unclear when this gene family evolved in plants. Members of this family encode transcription factors characterized by two domains, a zinc finger domain at the $\mathrm{N}$ terminus and a YABBY domain at the C terminus (Bowman and Smyth, 1999; Sawa et al., 1999a). The YABBY domain is similar in structure to the high mobility group (HMG) domain (Sawa et al., 1999a) and is necessary for DNA binding to occur (Kanaya et al., 2002). Four gene duplication events in this gene family have occurred prior to the diversification of the angiosperms (Bartholmes et al., 2012), leading to genes with both novel and redundant functions.

In angiosperms, $Y A B B Y$ genes have important roles in laminar expansion of lateral organs as well as in reproductive organ development and other processes. The YABBY gene family has six members in Arabidopsis thaliana. FILAMENTOUS FLOWER (FIL), YABBY2, and YABBY3 are expressed in the abaxial domain of lateral organs and act redundantly to specify abaxial cell fate and ultimately to promote laminar outgrowth (Siegfried et al., 1999); FIL is also required for normal inflorescence and flower development (Chen et al., 1999; Sawa et al., 1999b). CRABS CLAW (CRC) and INNER NO OUTER (INO) have specialized functions in reproductive organ development: $C R C$ is expressed in carpels and nectaries and is necessary for gynoecium elongation and nectary development (Alvarez and Smyth, 1999; Bowman and Smyth, 1999), and INNER NO OUTER (INO) is expressed in the abaxial domain of the outer integument and is required for normal outer integument development (Baker et al., 1997; Villanueva et al., 1999).

Studies in other eudicot species suggest that the abaxial expression pattern and specific function in promoting laminar outgrowth may be conserved across the eudicot lineage. In tomato, $\operatorname{LeYAB} B$ is expressed abaxially and may also specify abaxial identity (Kim et al., 2003). In Antirrhinum majus, GRAMINIFOLIA (GRAM), a FIL-like gene, and PROLONGATA $(P R O L)$, a $Y A B B Y 5$-like gene, are expressed abaxially and promote laminar outgrowth (Golz et al., 2004). GRAM has also been shown to be involved in the control of floral organ initiation and identity (Navarro et al., 2004). TmFIL in Tropaelum majus and SrGRAM in Streptocarpus rexii, both FIL-like genes, are also expressed abaxially (Gleissberg et al., 2005; Tononi et al., 2010).
Studies in monocots, which have focused mainly on grass species (Poales), have demonstrated varied expression patterns leading to a diversity of proposed functions. In Zea mays (maize), the FIL/YAB3-like genes ZYB9 and ZYB14 are expressed adaxially and may play a role in lateral outgrowth (Juarez et al., 2004). In contrast, OsYABBY1, a YABBY2-like gene from Oryza sativa (rice), is expressed in precursor cells that give rise to abaxial sclerenchyma in the leaves, the mestome sheath in the large vascular bundle, and sclerenchymatous cells in the palea and lemma of the flower, and is thus proposed to specify differentiation of certain cell types in rice (Toriba et al., 2007). The FIL-like gene OsYABBY4 is expressed in meristems and in developing phloem and thus may be involved in vasculature development in rice (Liu et al., 2007). The gene AaCRC from Asparagus asparagoides is expressed in the abaxial region of the ovary wall and leaf phloem (Nakayama et al., 2010), while $C R C / D L$ orthologs in Poales species are expressed throughout the carpel and in the central region of the leaf and may specify carpel identity and midrib formation (Yamaguchi et al., 2004; Ishikawa et al., 2009). It seems that divergence in expression (and possibly function) of $Y A B B Y$ genes has occurred more so in the monocot lineage than in the eudicot lineage.

Studies from the early diverging angiosperms show variation in expression as well. $A m b F 1$, a YABBY2 homolog from Amborella trichopoda, is expressed adaxially in all floral organs, the shoot apex, and leaves (Yamada et al., 2004). YABBY genes from Cabomba caroliniana (CcFIL, CcYAB5, CcINO, and

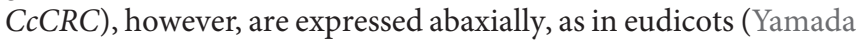
et al., 2011). In two Nymphaea species, CcINO is expressed in the outer epidermis of the outer integument, as is INO in Arabidopsis, but is also expressed in the inner integument and the tip of the nucellus (Yamada et al., 2003). AmbCRC from A. trichopoda is expressed in the abaxial carpel, maintaining a similar expression pattern to that observed in Arabidopsis (Fourquin et al., 2005).

Overall, abaxial expression seems to be conserved across eudicots, and YABBY function in controlling laminar outgrowth seems to be generally conserved across angiosperms, but shifts in expression have been observed, particularly in the monocots. Based on the expression patterns of $Y A B B Y$ genes across angiosperms, the strong expression of these genes in ectopic outgrowths on both abaxial and adaxial surfaces in polarity mutants, and the later timing of $Y A B B Y$ gene expression relative to that of other genes in the polarity gene network, Husbands et al. (2009) have proposed that the ancestral function of $Y A B B Y$ genes in angiosperms may have been to promote blade outgrowth at abaxial-adaxial boundaries.

The evolution of laminarity in the androecium and gynoecium contributes to diversity in floral morphology and the evolution of plant-pollinator interactions in the tropical monocot order Zingiberales (Specht et al., 2012). This group includes the four paraphyletic banana families (Musaceae, Strelitziaceae, Lowiaceae, and Heliconiaceae) and a monophyletic group of four ginger families (Cannaceae, Marantaceae, Zingiberaceae, and Costaceae). The banana lineages are characterized by five or six fertile stamens with radial filaments, and members of Heliconiaceae have one laminar staminode, while members of 
the ginger clade exhibit a reduction in fertile stamen number-to one in Zingiberaceae and Costaceae and to one-half (i.e., fertile stamen with a single theca) in Cannaceae and Marantaceaeand are characterized by laminar, petaloid staminodes and fertile stamens. Members of Zingiberaceae and Costaceae possess a novel petaloid structure, the labellum, formed from two or four (Zingiberaceae) or five (Costaceae) fused laminar staminodes. The staminodes and the labellum are likely to function in pollinator attraction, making up most of the floral display in terms of showiness, coloration, and symmetry (Specht et al., 2012). In Cannaceae, the gynoecium style is also laminar and is used for secondary pollen presentation: during flower development, pollen adheres to the lateral surface of the style and is then transferred to the bill of a hummingbird pollinator once the flower opens (Glinos and Cocucci, 2011).

The evolution of laminarity in different floral organs in the Zingiberales makes this group a useful system in which to investigate the evolution of this trait and the evolution of genes involved in the abaxial-adaxial polarity gene network. Results from a recent study on the evolution of stamen morphology in the Zingiberales (Almeida et al., 2014) implicated balanced expression of abaxial-adaxial polarity genes in the formation of laminar filaments in the ginger clade, while overexpression of a $Y A B B Y 2 / 5$ gene was implicated in the formation of radial filaments in Musa. Interestingly, the authors also demonstrated a similar gene expression pattern in Brassica rapa radial filaments, suggesting that $Y A B B Y 2 / 5$ genes are involved in the evolution of filament morphology in angiosperms (Almeida et al., 2014).

In order to better understand the evolution of the YABBY2 gene subfamily in the Zingiberales and its role in the evolution of laminarity in the style of Cannaceae, we isolated homologs of Arabidopsis YABBY2 from taxa across the order and performed phylogenetic and expression analyses to investigate the evolutionary history of the gene subfamily. We identified duplication events occurring prior to the diversification of the Zingiberales and more recent, lineage-specific duplication events within the order. These data, in combination with expression data from semi-quantitative RT-PCR, were used to describe a hypothesized role of YABBY2-like genes in the evolution of the laminar style. We also tested for selection along branches in the $Y A B B Y 2$ gene subfamily and looked for motifs characteristic of YABBY2-like genes.

\section{MATERIALS AND METHODS}

\section{Plant Material and cDNA Synthesis}

Floral buds of Zingiberales species (Table 1) were collected and immediately frozen in liquid nitrogen. Floral tissue was stored at $-80^{\circ} \mathrm{C}$ until RNA extraction. Floral organs of young flowers from Costus spicatus, Canna indica, and Musa basjoo were also dissected in order to extract organ-specific RNA. Organspecific material was immediately frozen in liquid nitrogen and stored at $-80^{\circ} \mathrm{C}$ until RNA extraction. RNA extractions were performed with Plant RNA Reagent (Life Technologies) according to Yockteng et al. (2013). RNA was treated with Turbo DNase (Ambion) and cDNA was synthesized from
$1.0 \mu \mathrm{g}$ of DNase-treated RNA using iScript reverse transcriptase and oligo(dT) primers following the manufacturer's protocol (Bio-Rad Laboratories). As a control, a cDNA synthesis reaction without reverse transcriptase was set up for each sample.

\section{Isolation of YABBY2 in the Zingiberales Sequence Retrieval}

Previously published sequences from across the YABBY gene family were retrieved from NCBI, with representatives from early diverging angiosperms, monocots, early diverging eudicots, core eudicots, and gymnosperms. YABBY sequences from NCBI were blasted against the genome of Musa acuminata (D'Hont et al., 2012) and against the whole flower transcriptomes of Costus spicatus, Canna indica, and Musa basjoo (unpublished; Roxana Yockteng, Ana M. R. Almeida, and Chelsea D. Specht)

TABLE 1 | Zingiberales taxa used in this study and obtained YABBY2-like sequences.

\begin{tabular}{|c|c|c|}
\hline Species & Location & $\begin{array}{l}\text { NCBI accession } \\
\text { numbers }\end{array}$ \\
\hline $\begin{array}{l}\text { Costus spicatus (Jacq.) } \\
\text { Sw. }\end{array}$ & 2002-127 (NMNH) & KT795168-KT795183 \\
\hline Canna indica L. & $\begin{array}{l}\text { 2011-777 (UC Specht } \\
\text { Lab) }\end{array}$ & KT795161 \\
\hline Musa acuminata Colla & 2002-075 (NMNH) & KT795221-KT795230 \\
\hline Musa basjoo Siebold & 89.0873 (UCBG) & KT795231-KT795238 \\
\hline $\begin{array}{l}\text { Zingiber officinale } \\
\text { Roscoe }\end{array}$ & $\begin{array}{l}\text { MB0876 (UC Specht } \\
\text { Lab) }\end{array}$ & KT795282-KT795284 \\
\hline Strelitzia sp. Banks & UC Specht Lab & KT795268-KT795281 \\
\hline $\begin{array}{l}\text { Marantochloa } \\
\text { leucantha (K. Schum.) } \\
\text { Milne-Redh. }\end{array}$ & L-80.0376 (HLA) & KT795214-KT795220 \\
\hline $\begin{array}{l}\text { Orchidantha siamensis } \\
\text { K. Larsen }\end{array}$ & L-91.0308 (HLA) & KT795240-KT795247 \\
\hline $\begin{array}{l}\text { Phrynium oliganthum } \\
\text { Merr. }\end{array}$ & L-96.0226 (HLA) & KT795248-KT795251 \\
\hline $\begin{array}{l}\text { Schumannianthus } \\
\text { virgatus (Roxb.) Rolfe }\end{array}$ & L-83.0899 (HLA) & KT795258-KT795267 \\
\hline $\begin{array}{l}\text { Heliconia pendula } \\
\text { Wawra }\end{array}$ & 71003-003 (McBryde) & KT795200-KT795207 \\
\hline $\begin{array}{l}\text { Kaempferia } \\
\text { rubromarginata (S. Q. } \\
\text { Tong) R. J. Searle }\end{array}$ & L-2003.0153 (HLA) & KT795211-KT795213 \\
\hline $\begin{array}{l}\text { Etlingera corneri Mood } \\
\text { and Ibrahim }\end{array}$ & L-91.0443 (HLA) & KT795184-KT795187 \\
\hline $\begin{array}{l}\text { Pleuranthodium helwigii } \\
\text { (K. Schum.) R. M. Sm. }\end{array}$ & L-99.0492 (HLA) & KT795252-KT795257 \\
\hline Globba laeta K. Larsen & L-92.0182 (HLA) & KT795188-KT795195 \\
\hline Heliconia sp. L. & UC Specht Lab & KT795208-KT795210 \\
\hline Canna jaegeriana Urb. & MB0854 (UC) & KT795162-KT795167 \\
\hline $\begin{array}{l}\text { Heliconia caribaea x } \\
\text { bihai Lam. and L. }\end{array}$ & $\begin{array}{l}\text { MB0862 (UC Specht } \\
\text { Lab) }\end{array}$ & KT795196-KT795199 \\
\hline Musa textilis Née & 1682/77 (NYBG) & KT795239 \\
\hline
\end{tabular}

HLA, Lyon Arboretum, Oahu, Hawaii; McBryde, McBryde Botanical Garden, Kauai, Hawaii; UCBG, University of California Botanical Garden; UC, University of California; NMNH, Smithsonian Greenhouses; NYBG, New York Botanical Garden. 
to retrieve $Y A B B Y$ genes from these taxa. We also used RNA-seq reads from the Monocot AToL (Angiosperm Tree of Life $)^{1}$ project to assemble transcriptomes from Costus pulverulentus, Canna indica, Curcuma roscoeana, Orchidantha fimbriata, Heliconia collinsiana, Zingiber spectabile, Strelitzia reginae, and Maranta leuconeura using Trinity r2013_08_14 (Grabherr et al., 2011). YABBY sequences were again blasted against these transcriptomes to retrieve $Y A B B Y$ s from these taxa. Source and accession numbers for all of the sequences used in this study are shown in Table S1.

\section{Primer Design}

The YABBY2 sequences from NCBI, the Musa acuminata genome, and the floral transcriptomes were aligned using the Geneious v5.6 algorithm (Drummond et al., 2012) and manually edited to further refine the alignment. This multiple sequence alignment was used to design forward and reverse primers at conserved sites of the gene-at the N-terminal end of the zinc finger domain and the C-terminal end of the YABBY domain, respectively-and these primers were used to amplify $Y A B B Y 2$ from taxa across the Zingiberales. Zingiberales sequences then obtained through cloning were edited and added to the alignment, along with the transcriptome contigs from MonAToL, and a preliminary tree was used to design cladespecific primers of Zingiberales sequences falling in the YABBY2 clade in order to amplify more sequences from Zingiberales taxa in each clade. All primers used in this study are found in Table S2.

\section{Cloning of $Y A B B Y 2$ in the Zingiberales}

$Y A B B Y 2$ orthologs from taxa across the eight families in the Zingiberales (Table 1) were amplified using $1.0 \mu \mathrm{l}$ of cDNA diluted 1:50 in water, $0.3 \mu \mathrm{mol}$ of each primer, and Phire II Hot Start DNA Polymerase (Thermo Scientific) as follows: $5 \mathrm{~min}$ at $98^{\circ} \mathrm{C}$ for initial denaturing; 40 cycles of $5 \mathrm{~s}$ at $98^{\circ} \mathrm{C}$ for denaturing, $5 \mathrm{~s}$ at a primer-specific annealing temperature for annealing, and $20 \mathrm{~s}$ at $72^{\circ} \mathrm{C}$ for extension; and $1 \mathrm{~min}$ at $72^{\circ} \mathrm{C}$ for final extension. PCR products were cloned into pJET1.2 blunt cloning vector (Thermo Scientific). Sequencing was performed using BigDye v3.1 on a 3730 Applied Biosystems DNA analyzer at the Museum of Vertebrate Zoology Evolutionary Genetics Laboratory at UC Berkeley.

\section{Phylogenetic Analyses}

All of the Zingiberales sequences obtained in this study and $Y A B B Y$ sequences retrieved from NCBI, the Musa acuminata genome, the floral transcriptomes listed above, and the MonAToL transcriptomes listed above were aligned using the Geneious v5.6 algorithm and manually edited using a codonpreserving approach to further refine the alignment. Unalignable regions outside of the zinc finger and YABBY domains were removed, resulting in a truncated alignment used for further analyses. The final truncated alignment was composed of a 132 nucleotide-long conserved region including the zinc finger domain and a 168 nucleotide-long conserved region including

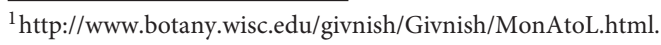

the YABBY domain (Figure S1), with a total alignment length of 300 nucleotides.

jModeltest 2.1.1 (Darriba et al., 2012) was used for selection of the best fit model of nucleotide evolution, and indicated the TVMef $+\mathrm{I}+\mathrm{G}$ model as most appropriate for the given nucleotide alignment according to the Bayesian information criterion (BIC).

Bayesian inference was used to infer a phylogeny using MrBayes 3.2.2 (Ronquist and Huelsenbeck, 2003) in the Cipres Science Gateway ${ }^{2}$ using the model specified above. The MCMC was run for 10,000,000 generations and the output files were analyzed in Tracer (Rambaut et al., 2014) to check for convergence of the two chains.

A maximum likelihood phylogeny with 100 bootstrap replicates was reconstructed using PhyML 3.0 (Guindon et al., 2010) using the model specified in jModeltest.

\section{Selection Tests}

SLAC, FEL, and branch-site REL selection tests were implemented in HYPHY (Kosakovsky Pond et al., 2005; Kosakovsky Pond and Frost, 2005a) on the Datamonkey webserver (Kosakovsky Pond and Frost, 2005b) to identify sites under positive or negative (purifying) selection across the truncated multiple sequence alignment. The SLAC analysis was run using the same 300 nucleotide-long truncated multiple sequence alignment used for phylogenetic analyses, containing 133 sequences (Figure S1), and the maximum likelihood tree reconstructed using PhyML (Figure 2). The nucleotide model used was chosen by a Datamonkey model selection analysis. The global $\mathrm{dN} / \mathrm{dS}$ value was estimated and ambiguities were averaged, and the significance level was set to 0.05. The FEL analysis was run using the same alignment, nucleotide model, and ML tree, and the significance level was set to 0.05. A branch-site REL analysis was also implemented in HYPHY using a reduced multiple sequence alignment containing 72 sequences representing all major clades in the $Y A B B Y$ gene tree, and a ML tree generated in PhyML.

We also tested for positive selection on codons along 9 branches of major clades in the $Y A B B Y 2$ subfamily, in separate analyses for each branch, using branch-site model A in codeml (model $=2$ and NS sites $=2$ ) implemented in PAML (Yang, 2007). For each analysis, a likelihood ratio test (LRT) was used to determine if the difference between the likelihood scores of the alternative model (model $\mathrm{A}$, in which $\omega$ for the branch of interest is estimated and the background $\omega$ is set to 0 ) and the null model (in which the distribution of $\omega$ is set to 1 ) was significantly different (degrees of freedom $=1$ ). For each LRT, a $p$-value of 0.05 or less was required for results to be considered significant.

\section{Motif Identification Using MEME}

MEME (Multiple Em for Motif Elicitation) version 4.10.0 (Bailey and Elkan, 1994) was used to identify ungapped motifs in translated YABBY2 protein sequences. Since this analysis does not require an alignment of sequences to identify conserved motifs, it could be used to search for motifs outside of the alignable regions. The "Normal" mode of motif discovery was

${ }^{2}$ http://www.phylo.org/portal2/. 
used to search for motifs within the default width range (6-50 amino acids), and the site distribution for motifs was set to zero or one occurrence per sequence.

\section{Semi-Quantitative RT-PCR}

Reverse transcription PCR was used to determine presence or absence of expression of copies of YABBY2 in the total flower, floral organs, and young leaves of Costus spicatus, Musa basjoo, and Canna indica. RNA extraction and cDNA synthesis were as described above. cDNA was synthesized from C. spicatus total flower, sepals, petals, labellum, theca, filament, gynoecium, style, and young leaves; M. basjoo total flower, free petal, floral tube (fused sepals and petals), theca, filament, gynoecium, style, and young leaves; and C. indica total flower, sepals, petals, petaloid fertile stamen, theca, staminodes, gynoecium, style, and young leaves. Primers for each gene copy were designed across intron-exon boundaries to avoid amplification of trace contaminating gDNA. All RT-PCRs were performed using $1.0 \mu \mathrm{l}$ of cDNA diluted 1:20 in water, $0.3 \mu \mathrm{mol}$ of copy-specific forward and reverse primers (Table S2), and Phire Hot-Start II DNA Polymerase (Thermo Scientific) as follows: $5 \mathrm{~min}$ at $98^{\circ} \mathrm{C}$ for initial denaturing followed by $35-37$ cycles of $5 \mathrm{~s}$ at $98^{\circ} \mathrm{C}$ for denaturing, $5 \mathrm{~s}$ at a primer-specific annealing temperature for annealing, and $25 \mathrm{~s}$ at $72^{\circ} \mathrm{C}$ for elongation. The PCR products were run on $1 \%$ agarose gels.

Additionally, semi-quantitative RT-PCR was used to gauge relative levels of expression of YABBY2 copies in the styles of Costus spicatus, Musa basjoo, and Canna indica. For each gene copy, five different reactions were run with different numbers of cycles $(25,27,30,32$, and 35 cycles), while all other conditions were as described above. The PCR products were run on $1 \%$ agarose gels.

Products from the RT-PCRs were sequenced to confirm that the products being amplified were the targeted gene copy. At least three technical replicates were run for each reaction. ACTIN was used as an endogenous control; ACTIN primers used for each species are included in Table S2.

\section{RESULTS}

We obtained sequences for YABBY2-like genes from representative taxa across the Zingiberales in order to investigate the evolution of this gene subfamily and its potential role in the evolution of floral morphology in this order, particularly the evolution of laminarity in the gynoecium. To obtain sampling across the Zingiberales phylogeny, at least one taxon was sampled from each family. A total of 124 sequences were obtained from 19 taxa through cloning (Table 1). The sequences obtained in this study were blasted against the NCBI database to confirm that they do indeed blast to previously published YABBY2 genes, and consensus sequences were made for sequences from the same taxa that shared at least $98 \%$ identity. Six contigs from the whole flower transcriptomes of Costus spicatus and Musa basjoo (unpublished; Roxana Yockteng, Ana M.R. Almeida, and Chelsea D. Specht) and four contigs from transcriptomes of Canna indica and Costus pulverulentus from MonAToL were also used in analyses. All sequences obtained in this study have been deposited in GenBank (KT795161-KT795284).

Almost all sequences used in phylogenetic analyses contain the zinc finger and YABBY domains characteristic of genes of the $Y A B B Y$ gene family; some sequences are missing some of the zinc finger domain and/or the YABBY domain due to primer design limitations. Sequences from at least one taxon from each family were included in the final alignment used for phylogenetic analyses. This alignment also included sequences from other monocots, core eudicots, early diverging eudicots, early diverging angiosperms, and gymnosperms.

\section{Evolution of YABBY2-Like Genes in the Zingiberales}

The maximum likelihood reconstruction of the $Y A B B Y$ gene family is shown (Figure 2). Bootstrap values are shown at tree nodes, with bootstrap values greater than 50 in bold. When rooted with the gymnosperm group, the topology recovered is consistent with one of the previously proposed topologies for this gene family (Bartholmes et al., 2012), with the YABBY2 and YABBY5 subfamilies sister to the INO, FIL, and CRC subfamilies. The FIL and CRC subfamilies are sister to each other, and the INO subfamily is sister to both. The YABBY2 and YABBY5 subfamilies form sister clades.

Within the YABBY2 subfamily, the YABBY2-like sequence from the early diverging angiosperm Amborella trichopoda is sister to all other YABBY2-like sequences from eudicots and monocots. The eudicot YABBY2-like sequences form a clade sister to a clade of monocot YABBY2-like sequences. The monocot YABBY2-like clade is further divided into two sister clades: one of these clades includes a clade of Poales sequences sister to three separate clades of Zingiberales sequences (ZinYAB2-1, ZinYAB2-2, and ZinYAB2-3), and the other monocot clade is composed of a clade of non-Zingiberales monocot sequences (including Poales sequences) sister to a clade of Zingiberales sequences (ZinYAB2-4) and one Elaeis guineensis sequence. Each of the Zingiberales clades includes at least one sequence from each family in the Zingiberales, with the exception of $\operatorname{Zin} Y A B 2-1$, which does not have any sequences from Cannaceae. The Poales clade, the ZinYAB2-1 clade, and the (Elaeis guineensis + ZinYAB2-4) clade are moderately well supported by the bootstrap analysis (with bootstrap values of 93 , 85 , and 68 , respectively); bootstrap values for other clades are low, but have high or moderately high posterior probabilities in a Bayesian analysis (Figure S2).

\section{Expression of ZinYAB2 Genes}

Reverse transcription PCR was used to evaluate the presence and absence of expression of YABBY2-like genes in the floral organs and young leaves of Canna indica (Cannaceae), Costus spicatus (Costaceae), and Musa basjoo (Musaceae). Semi-quantitative RTPCR was also used to gauge relative expression of YABBY2like genes in the styles of these species. These taxa span the Zingiberales phylogeny and were chosen to represent the diverse floral morphology found in the order (Figure 1). Differential expression between species could indicate changes in function of YABBY2-like genes in the development of floral organs. 
Expression profiles are represented (Figure 3) and gel images for the RT-PCRs can be found in the Supplementary data (Figure S3).

In the Zingiberales, YABBY2-like genes show differential expression between gene copies, between species, and across floral organs and leaves (Figure 3). In Musa basjoo, ZinYAB21 is expressed in all floral organs and in young leaves. In Costus spicatus, ZinYAB2-1a and ZinYAB2-1b are expressed in all floral organs except for stamen theca and style; ZinYAB2$1 b$ is present in C. spicatus young leaves while ZinYAB2-1a is absent (Figure 3). In C. indica, ZinYAB2-1 has been lost or has not been recovered in this study. ZinYAB2-2 is expressed in all floral organs in both $M$. basjoo and C. spicatus, while in $C$. indica this copy is expressed in petal, petaloid filament, staminode, gynoecium, and young leaves and is absent from sepal, theca, and style (Figure 3). In M. basjoo, there are three copies of $\operatorname{Zin} Y A B 2-3$, resulting from duplication events in the Musaceae lineage (Figure 2): $\operatorname{ZinYAB2-3a}$ is expressed in all floral organs except filament, $\operatorname{Zin} Y A B 2-3 b$ is expressed in all floral organs except free petal and filament, and ZinYAB2-3c is expressed in all floral organs (Figure 3). $\operatorname{ZinYAB2}-3 c$ is the only ZinYAB2-3 copy in M. basjoo that is expressed in young leaves. ZinYAB2-3 in both C. spicatus and C. indica is expressed in young leaves and in all floral organs except theca. In $M$. basjoo, ZinYAB2-4 is expressed in free petal and floral tube and is absent from filament, theca, gynoecium, style, and young leaves. In C. spicatus, ZinYAB2-4 is present in total flower (as confirmed by three technical replicates) but was not amplified by RT-PCR for any floral organs; it is possible that this copy is so lowly expressed that it could not be amplified from floral organ tissue. In C. indica, ZinYAB2-4 is expressed in sepal, petal, and theca, and is absent from filament, staminode, gynoecium, style, and young leaves. ZinYAB2-4 seems to be expressed only in the flower, since there is no expression in leaves (Figure 3).
Interestingly, ZinYAB2-4 is absent from filament and gynoecium in all species considered; expression of this copy has diverged the most from that of the other YABBY2-like gene copies in the Zingiberales.

Across the Zingiberales, there is a pattern of reduction in the number of $Y A B B Y 2$-like gene copies expressed in the style: five gene copies are expressed in M. basjoo (ZinYAB2-1, ZinYAB2-2, Zin YAB2-3a, ZinYAB2-3b, and ZinYAB2-3c), two in C. spicatus (ZinYAB2-2 and ZinYAB2-3), and one in C. indica (ZinYAB2-3). Musa basjoo and C. spicatus have the same style morphologyradial-but $Y A B B Y 2$-like expression between styles in these two species differs not only in the number of gene copies expressed, but also in the overall expression level of YABBY2-like genes (Figure 3). One copy, ZinYAB2-2, is expressed in M. basjoo and C. spicatus styles but is absent from the style of $C$. indica.

\section{Selection in ZinYAB2 Genes}

Of the 100 sites tested, 87 sites were found to be under negative selection using the SLAC method and 90 sites were found to be under negative selection using the FEL test $(p=0.05)$. Neither test identified sites under positive selection. The branchsite REL analysis indicated that no branches are under episodic diversifying selection $(p \leq 0.05)$.

Of the nine branches of major clades in the $Y A B B Y 2$ subfamily that were tested in codeml, none had codons that were found to be under positive selection once likelihood ratio tests were used to evaluate significance.

\section{A Novel Motif Found in Monocot YABBY2-Like Genes}

MEME identified one motif that is conserved across most monocot $Y A B B Y 2$-like sequences. It is 15 residues in width and occurs before the YABBY domain, in-between the zinc finger and

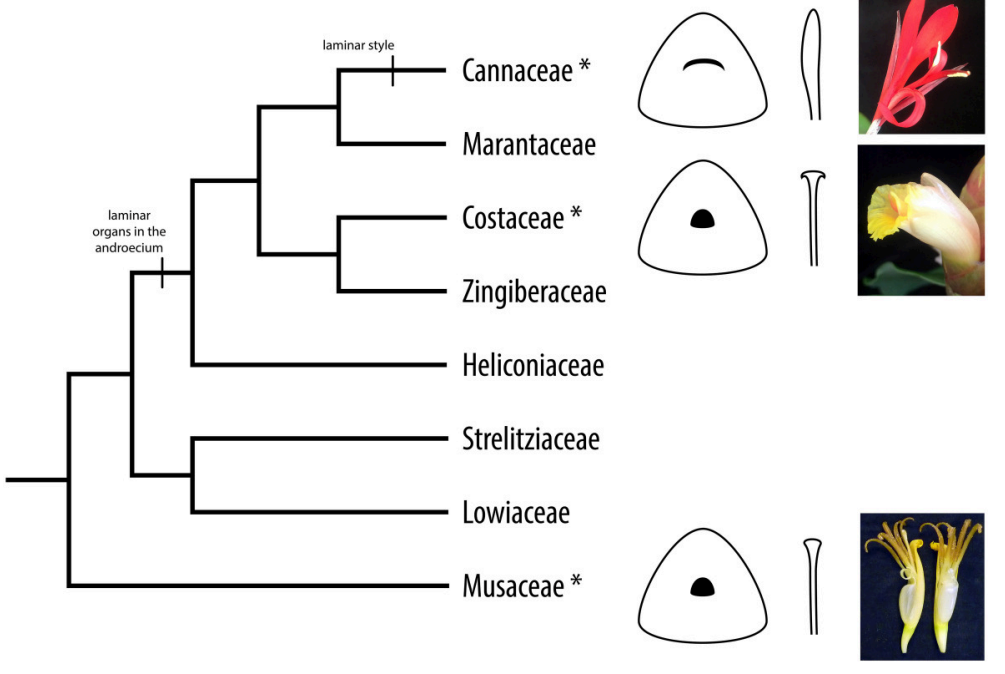

FIGURE 1 | Cladogram of the Zingiberales. Asterisks denote taxa used for expression analyses in this study. Evolution of laminar organs is indicated: flowers in Heliconiaceae have radial filaments and one laminar staminode; flowers in the ginger clade (Cannaceae, Marantaceae, Zingiberaceae, and Marantaceae) have laminar staminodes and fertile stamens - with a staminodial labellum in Zingiberaceae and Costaceae; and flowers in Cannaceae have a laminar style. Style morphology for Cannaceae, Costaceae, and Musaceae is illustrated and flowers from representative species are shown. 


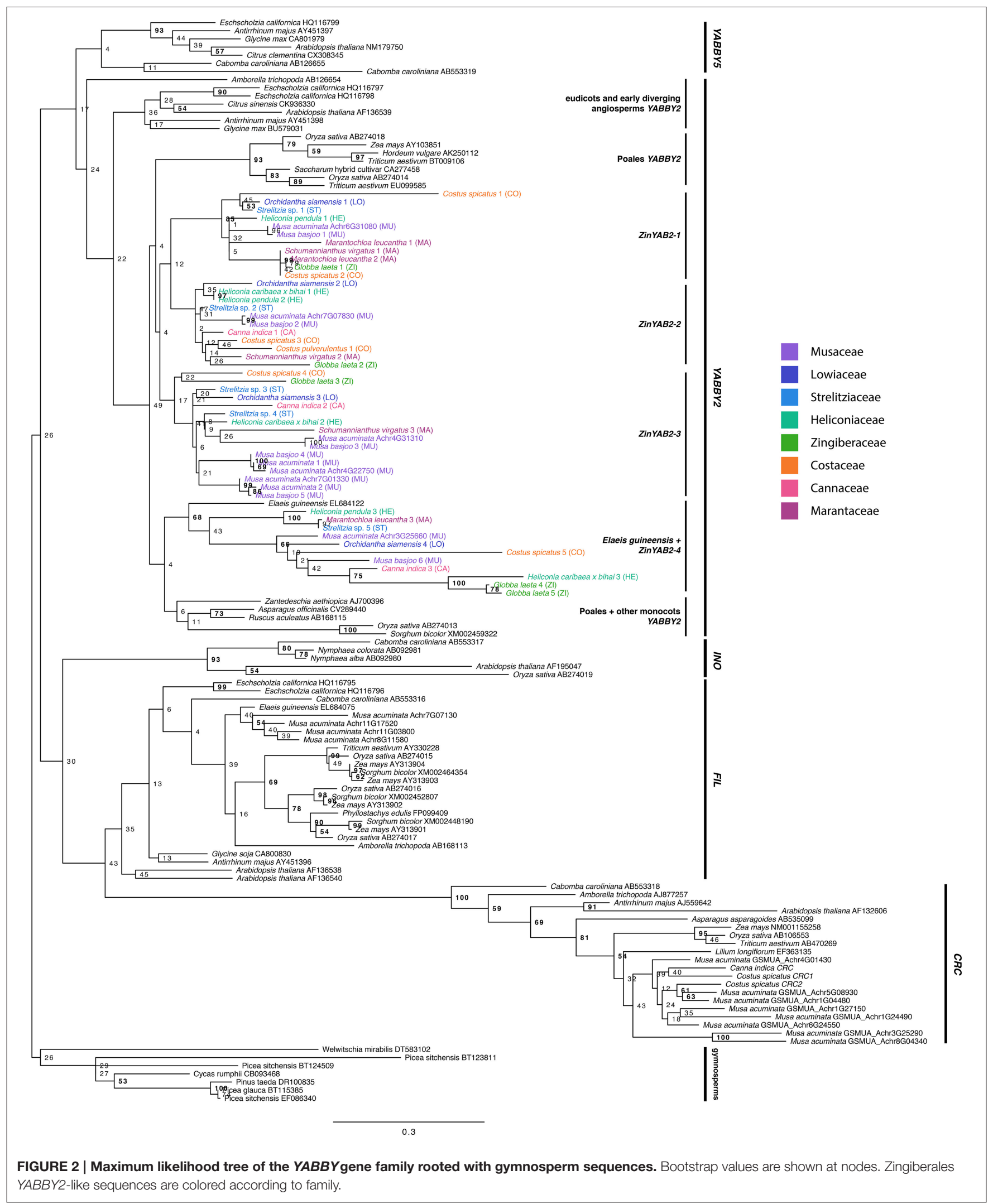




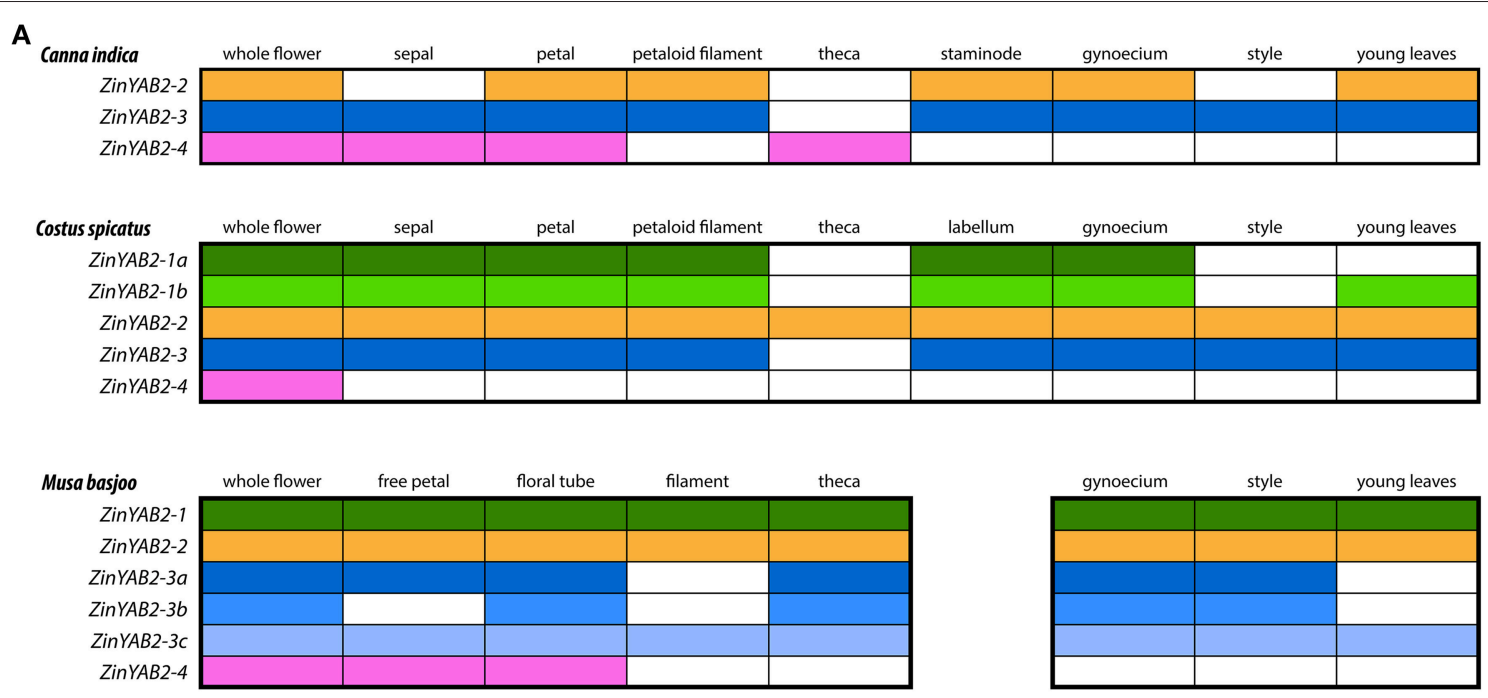

B

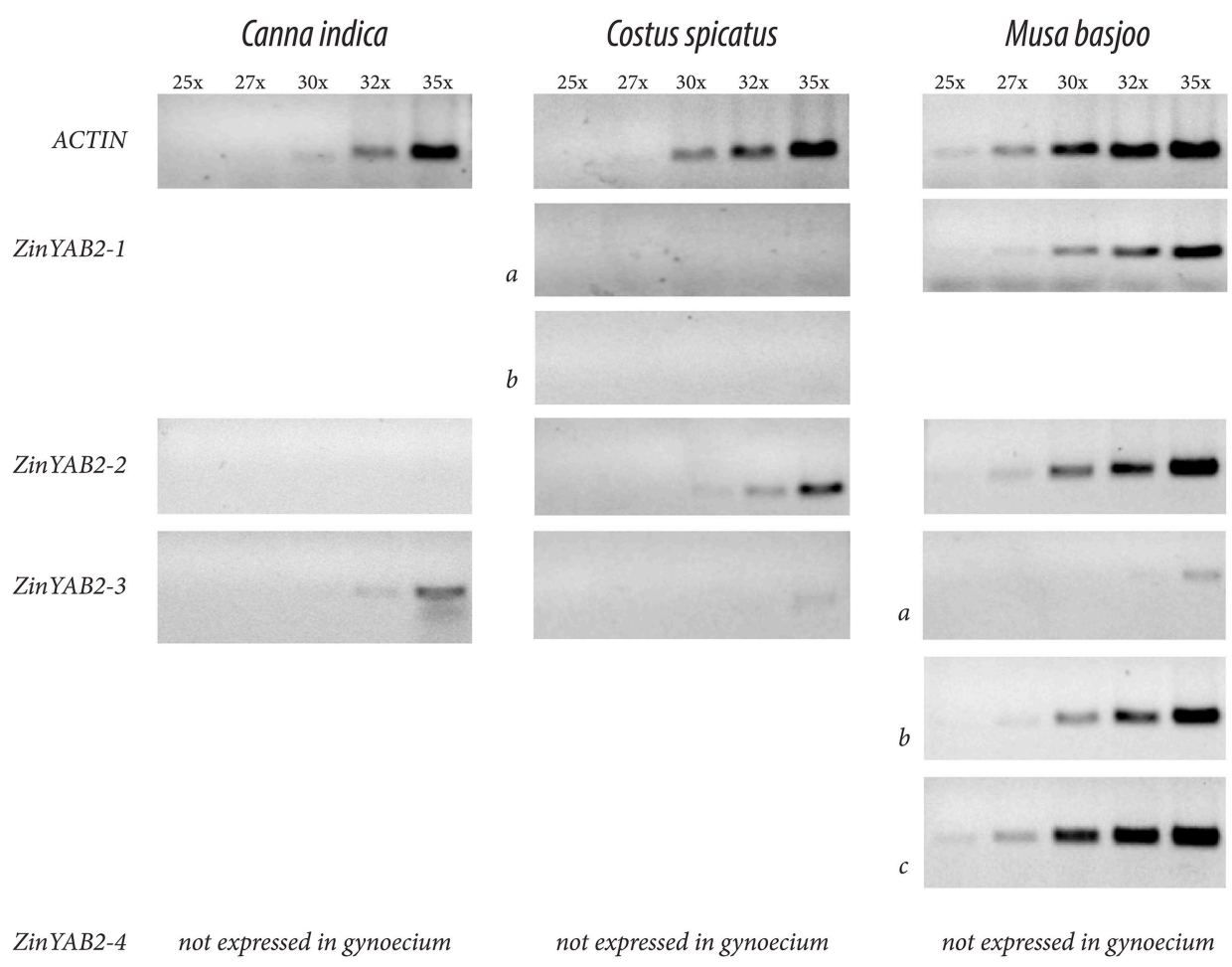

FIGURE 3 | Summary of RT-PCR results. (A) Summary of RT-PCR results from Canna indica, Costus spicatus, and Musa basjoo. Each color corresponds to a different copy of ZinYAB2; color indicates presence and lack of color indicates absence of the gene copy. (B) Summary of semi-quantitative RT-PCR results from flower styles of these taxa, showing results after 25, 27, 30, 32, and 35 cycles.

YABBY domains (Figure 4). Monocot YABBY2-like sequences that were identified as lacking the motif, when examined by eye, have some sequence similarity in this region, but have indels that make the conservation unrecognizable in the MEME analysis (which only recognizes ungapped motifs) or have amino acid changes that reduce similarity. This motif is not found in nonmonocot YABBY2-like genes or in other YABBY subfamilies.

\section{DISCUSSION}

The Zingiberales is an order of tropical monocots that possesses a wide diversity of floral forms. This diversity can in part be attributed to the evolution of laminarity in the androecium and gynoecium. Members of the banana lineages (Musaceae, Heliconiaceae, Strelitziaceae, and Lowiaceae) 


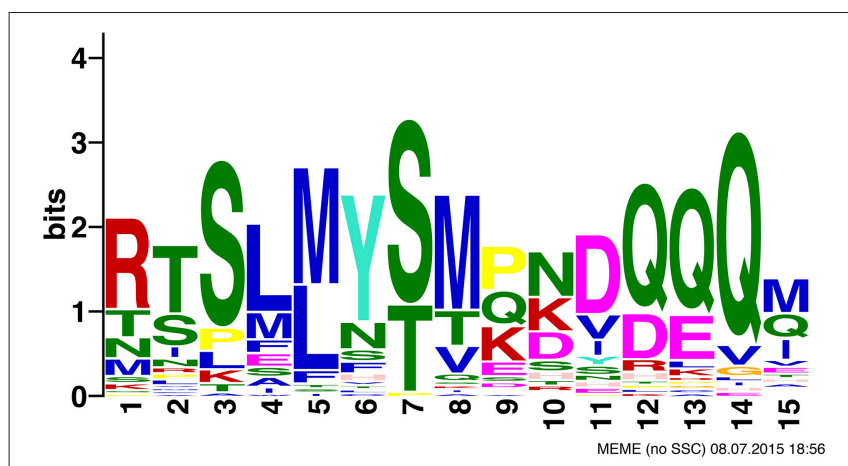

FIGURE 4 | MEME results showing a novel motif found in monocot YABBY2-like sequences.

possess radial filaments, but taxa in Heliconiaceae have single laminar staminodes. The ginger clade (Cannaceae, Marantaceae, Costaceae, and Zingiberaceae) is characterized by laminar, petaloid staminodes and stamens. Staminodes in species of Costaceae (5) and Zingiberaceae (2 or 4) are fused to form a novel laminar structure called the labellum. In Cannaceae, the gynoecium style has evolved to be laminar as well; all other families in the Zingiberales possess radial styles. Style laminarity in Cannaceae has functional importance because it facilitates secondary pollen presentation in this group: before a flower opens, pollen is transferred from the anthers to the laminar style. From there, the pollen is transferred to the bill of a hummingbird pollinator after the flower opens (Glinos and Cocucci, 2011).

In order to better understand the evolution of the laminar style in Cannaceae, we are interested in elucidating the molecular mechanisms involved in its development. In this study we have chosen to focus on one gene subfamily in particular, the YABBY2 gene subfamily. The $Y A B B Y$ gene family encodes transcription factors involved in the abaxial-adaxial polarity molecular network responsible for laminar expansion of lateral organs (and, conversely, radialization without laminar expansion). The $Y A B B Y 2$ gene subfamily was chosen because previous work has implicated the over-expression of a YABBY2-like gene as a mechanism for radialization in the filament of Musa acuminata and Brassica rapa (Almeida et al., 2014). To see if a similar mechanism may be involved in the evolution of the laminar style in Cannaceae, we have investigated the evolution of YABBY2like genes in the Zingiberales and considered gene duplications and subsequent shifts in gene expression as a mechanism for morphological evolution.

\section{Evolution and Diversification of YABBY2 in the Zingiberales}

The YABBY2-like genes from monocots form two clades, each with a non-Zingiberales monocot clade (including Poales sequences) sister to a Zingiberales clade. This suggests that there may have been a gene duplication event prior to the divergence of the Poales and Zingiberales; however, more in-depth efforts to isolate $Y A B B Y 2$-like genes from Poales species must be made to test this hypothesis. In one of these two monocot clades, the Zingiberales sequences are further divided into three separate clades, each with sequences from taxa from each the eight families in the Zingiberales-with the exception of ZinYAB21 , which lacks representation from Cannaceae-suggesting that two additional gene duplication events occurred in this gene lineage before the diversification of the Zingiberales. Due to our extensive efforts to design ZinYAB2-1-specific primers and to clone ZinYAB2-1 from Canna, we believe that ZinYAB21 has likely been lost in Cannaceae, or it could not be isolated due to rapid sequence divergence. A copy of ZinYAB2 was not found in either of the analyzed transcriptomes from Canna.

More recent gene duplication events have occurred within the Zingiberales. Two duplication events have occurred in Musaceae in the ZinYAB2-3 lineage, as is evidenced by Musa acuminata and Musa basjoo sequences falling sister to each other in familyspecific clades. Analysis of the Musa acuminata genome using the Genome Evolution analysis tool $(\mathrm{GeVo})$ from the CoGe platform (Lyons and Freeling, 2008) shows that two of these gene copies, ZinYAB2-3a and ZinYAB2-3c, are located in syntenic regions on chromosomes 4 and 7 and are likely alpha paralogs from a whole genome duplication event (Figure 5). The third Musa paralog, ZinYAB2-3b, may be from an older or local duplication event.

Duplication events in other Zingiberales families may have also occurred. The two Globba laeta (Zingiberaceae) ZinYAB24 sequences are recovered as being most closely related to each other and share $87.9 \%$ identity, while the two Costus spicatus (Costaceae) ZinYAB2-1 sequences are recovered as being more closely related to sequences from other species than to each other and share only $66.9 \%$ identity. This latter case is also true for the two Marantochloa leucantha (Marantaceae) ZinYAB2-1 sequences and the two Strelitzia sp. (Strelitziaceae) ZinYAB2-3 sequences, which share $73.5 \%$ identity and $86.9 \%$ identity, respectively. Based on sequence identity, these pairs of sequences could be allelic variants, or they could be the result of recent duplication events in these lineages.

\section{A Novel Motif Found in Monocot YABBY2-Like Sequences}

The monocot $Y A B B Y 2$-like motif identified in the MEME analysis could be an artifact of the shared evolutionary history of these sequences, or it could be biologically relevant. Functional tests need to be performed to test whether this motif is biologically relevant and whether it results in new or altered molecular interactions for monocot $Y A B B Y 2$-like sequences. Regardless, this motif could be helpful to identify $Y A B B Y 2$-like genes from monocots in future analyses, when it is difficult to obtain support for placement of genes in the $Y A B B Y$ gene family due to the conservation in the alignable regions (the zinc finger and YABBY domains). 


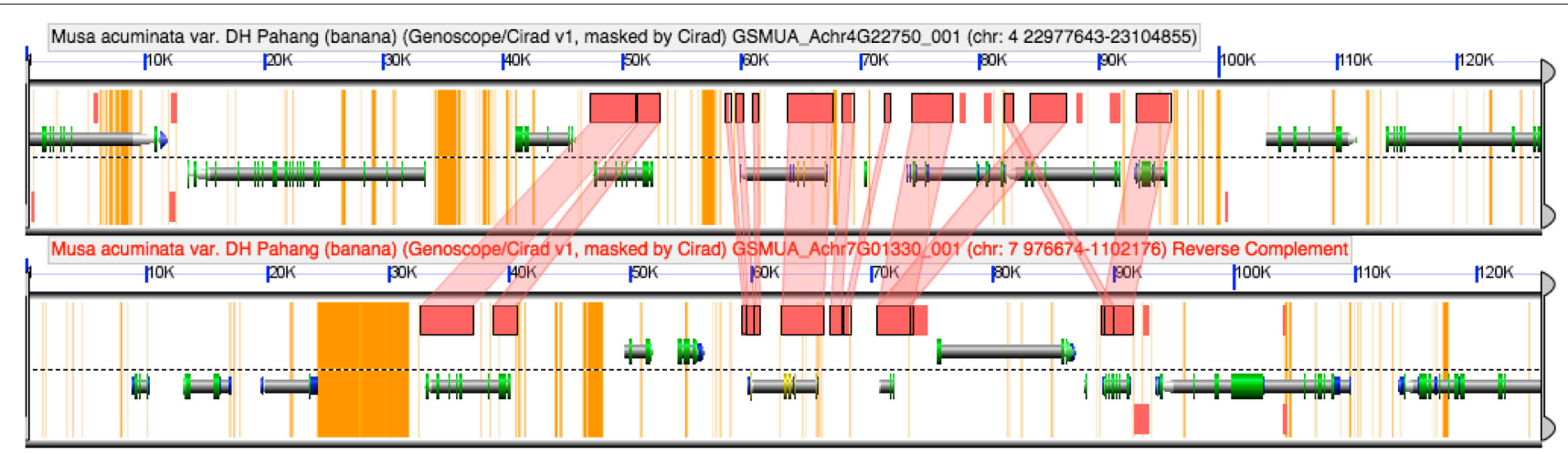

FIGURE 5 | COGE browser view of a portion of the Musa acuminata genome. ZinYAB2-3a and ZinYAB2-3c from Musa are found in syntenic regions in the Musa acuminata genome, suggesting that they are alpha paralogs from a recent whole genome duplication.

\section{Expression Profiles Support Proposed Evolutionary Relationships for ZinYAB2 Gene Copies}

The absence of ZinYAB2-4 from filament and gynoecium in C. indica, C. spicatus, and M. basjoo suggests that this copy is not involved in the gene network underlying laminar expansion (or radialization) in reproductive organs. The overall floral expression profiles of ZinYAB2-1, ZinYAB2-2, and ZinYAB2-3 are more similar to each other than to that of ZinYAB2-4, which has a more restricted expression profile. These data are consistent with the hypothesis that ZinYAB2-1, 2-2, and 2-3 arose from two subsequent Zingiberales-specific duplications, and that the ancestor of these three copies is sister to ZinYAB2-4 (and thus ZinYAB2-4 is more distantly related to the other three ZinYAB2 copies than they are to one another).

\section{A Potential Role of YABBY2-Like Genes in the Evolution of the Laminar Style in Cannaceae}

It has previously been proposed that balanced expression of genes involved in the abaxial-adaxial polarity network facilitates laminar expansion, with evidence for gene expression imbalance, through high expression of a $Y A B B Y 2$-like gene, as a mechanism for radialization in Musa acuminata and Brassica rapa filaments (Almeida et al., 2014). Musa basjoo and C. spicatus share a radial style morphology, but have different levels of expression and copy number of $Y A B B Y 2$-like genes. It is possible that there is a threshold for total YABBY2 expression in the style at which the abaxial-adaxial polarity gene network is in balance and promotes laminar expansion, and above which the gene network is imbalanced, leading to radialization. Loss of expression of ZinYAB2-2 (and, possibly, the loss of ZinYAB2-1) in the style of $C$. indica may have reduced total $Y A B B Y 2$-like gene expression to be in balance with other genes in the gene regulatory network, and thus facilitated the shift to laminar morphology in the style of $C$. indica.

Laminar expansion of the style is a derived trait in Cannaceae, and evolved separately from laminar expansion in stamens and staminodes (in Heliconiaceae and the ginger clade) and laminar expansion in sepals and petals. Perhaps the mechanism of balanced expression for laminar expansion is shared across different floral organs, but the absolute levels of expression required for laminar expansion differ in different organs; this would explain why expression differs between the laminar style and other laminar floral organs (sepal, petal, petaloid filament, and staminode) in C. indica.

\section{Future Directions}

To better understand the molecular mechanisms underlying the evolution of the laminar style in the Zingiberales, in situ hybridization experiments are needed to fully characterize the exact locations of $Y A B B Y 2$-like gene expression during development, and more gene evolution studies and expression analyses for other genes involved in the abaxial-adaxial gene regulatory network should be done. Gene knockdown (virus-induced gene silencing) or gene knockout (CRISPR) experiments are also needed to test the hypotheses proposed here about the functions of YABBY2-like genes in Zingiberales floral morphology. By using the evolution of style laminarity in Cannaceae as a case study, we can elucidate the mechanisms underlying the evolution of novel laminar (or radial) morphology in lateral organs. In addition, $Y A B B Y$ genes in gymnosperms have yet to be studied, and our understanding of the evolution of this gene family and thus the evolution of the abaxial-adaxial gene regulatory network would benefit greatly from gene characterization and expression studies in gymnosperms and pteridophytic vascular plants.

\section{AUTHOR CONTRIBUTIONS}

KM, RY, AA, and CS contributed with conceptual and experimental design. KM contributed to data collection and analysis and drafted the manuscript. RY and AA contributed to data analysis and manuscript editing. CS edited the manuscript and provided financial support. All authors read and approved the final manuscript. 


\section{ACKNOWLEDGMENTS}

This work was supported by an NSF CAREER award (IOS 0845641) to CS, an NSF Doctoral Dissertation Improvement Grant (DEB 1110461) to CS and AA, and a UC Berkeley College of Natural Resources student-initiated Sponsored Projects for Undergraduate Research (SPUR) award to KM. AA was supported by a Jovem Talento fellowship (CAPES/CNPq). The authors thank the members of the Specht Lab for discussion and

\section{REFERENCES}

Alvarez, J., and Smyth, D. R. (1999). CRABS CLAW and SPATULA, two Arabidopsis genes that control carpel development in parallel with AGAMOUS. Development 126, 2377-2386.

Bailey, T. L., and Elkan, C. (1994). "Fitting a mixture model by expectation maximization to discover motifs in biopolymers," in Proceedings of the Second International Conference on Intelligent Systems for Molecular Biology (Menlo Park, CA), 28-36.

Baker, S. C., Robinson-Beers, K., Villanueva, J. M., Gaiser, J. C., and Gasser, C. S. (1997). Interaction among genes regulating ovule development in Arabidopsis thaliana. Genetics 145, 1109-1124.

Bartholmes, C., Hidalgo, O., and Gleissberg, S. (2012). Evolution of the YABBY gene family with emphasis on the basal eudicot Eschscholzia californica (Papaveraceae). Plant Biol. 14, 11-23. doi: 10.1111/j.1438-8677.2011.00486.x

Bowman, J. L., Eshed, Y., and Baum, S. F. (2002). Establishment of polarity in angiosperm lateral organs. Trends Genet. 18, 134-141. doi: 10.1016/S01689525(01)02601-4

Bowman, J. L., and Smyth, D. R. (1999). CRABS CLAW, a gene that regulates carpel and nectary development in Arabidopsis, encodes a novel protein with zinc finger and helix-loop-helix domains. Development 126, 2387-2396.

Chen, Q., Atkinson, A., Otsuga, D., Christensen, T., Reynolds, L., and Drews, G. N. (1999). The Arabidopsis FILAMENTOUS FLOWER gene is required for flower formation. Development 126, 2715-2726.

Darriba, D., Taboada, G. L., Doallo, R., and Posada, D. (2012). jModelTest 2: more models, new heuristics and parallel computing. Nat. Methods 9, 772. doi: 10.1038/nmeth.2109

de Almeida, A. M., Yockteng, R., Schnable, J., Alvarez-Buylla, E. R., Freeling, M., and Specht, C. D. (2014). Co-option of the polarity gene network shapes filament morphology in the angiosperms. Nat. Sci. Rep. 4:6194. doi: 10.1038/srep06194

D’Hont, A., Denoeud, F., Aury, J. M., Baurens, F. C., Carreel, F., Garsmeur, O., et al. (2012). The banana (Musa acuminata) genome and the evolution of monocotyledonous plants. Nature 488, 213-217. doi: 10.1038/nature11241

Drummond, A. J., Ashton, B., Buxton, S., Cheung, M., Cooper, A., Duran, C., et al. (2012). Geneious v5.6. Available online at: http://www.geneious.com

Fourquin, C., Vinauger-Douard, M., Fogliani, B., Dumas, C., and Scutt, C. P. (2005). Evidence that CRABS CLAW and TOUSLED have conserved their roles in carpel development since the ancestor of the extant angiosperms. Proc. Natl. Acad. Sci. 102, 4649-4654. doi: 10.1073/pnas.0409577102

Gleissberg, S., Groot, E. P., Schmalz, M., Eichert, M., Kölsch, A., and Hutter, S. (2005). Developmental events leading to peltate leaf structure in Tropaeolum majus (Tropaeolaceae) are associated with expression domain changes of a YABBY gene. Dev. Genes Evol. 215, 313-319. doi: 10.1007/s00427-0050479-8

Glinos, E., and Cocucci, A. A. (2011). Pollination biology of Canna indica (Cannaceae) with particular reference to the functional morphology of the style. Plant Syst. Evol. 291, 49-58. doi: 10.1007/s00606-010-0379-x

Golz, J. F., Roccaro, M., Kuzoff, R., and Hudson, A. (2004). GRAMINIFOLIA promotes growth and polarity of Antirrhinum leaves. Development 131, 3661-3670. doi: 10.1242/dev.01221

Grabherr, M. G., Haas, B. J., Yassour, M., Levin, J. Z., Thompson, D. A., Amit, I., et al. (2011). Full-length transcriptome assembly from RNA-seq data without a reference genome. Nat. Biotechnol. 29, 644-652. doi: 10.1038/nbt.1883

Guindon, S., Dufayard, J. F., Lefort, V., Anisimova, M., Hordijk, W., and Gascuel, O. (2010). New algorithms and methods to estimate maximum-likelihood technical advice and Jennifer Bates for help with creating the figures.

\section{SUPPLEMENTARY MATERIAL}

The Supplementary Material for this article can be found online at: http://journal.frontiersin.org/article/10.3389/fpls.2015. 01106

phylogenies: assessing the peformance of PhyML 3.0. Syst. Biol. 59, 307-321. doi: 10.1093/sysbio/syq010

Husbands, A. Y., Chitwood, D. H., Plavskin, Y., and Timmermans, M. C. (2009). Signals and prepatterns: new insights into organ polarity in plants. Genes Dev. 23, 1986-1997. doi: 10.1101/gad.1819909

Ishikawa, M., Ohmori, Y., Tanaka, W., Hirabayashi, C., Murai, K., Ogihara, Y., et al. (2009). The spatial expression patterns of DROOPING LEAF orthologs suggest a conserved function in grasses. Genes Genet. Syst. 84, 137-146. doi: $10.1266 /$ ggs.84.137

Juarez, M. T., Twigg, R. W., and Timmermans, M. C. P. (2004). Specification of adaxial cell fate during maize leaf development. Development 131, 4533-4544. doi: 10.1242/dev.01328

Kanaya, E., Nakajima, N., and Okada, K. (2002). Non-sequence-specific DNA binding by the FILAMENTOUS FLOWER protein from Arabidopsis thaliana is reduced by EDTA. J. Biol. Chem. 277, 11957-11964. doi: 10.1074/jbc.M108889200

Kim, M., Pham, T., Hamidi, A., McCormick, S., Kuzoff, R. K., and Sinha, N. (2003). Reduced leaf complexity in tomato wiry mutants suggests a role for PHAN and KNOX genes in generating compound leaves. Development 130, 4405-4415. doi: 10.1242/dev.00655

Kosakovsky Pond, S. L., and Frost, S. D. W. (2005a). Not so different after all: a comparison of methods for detecting amino acid sites under selection. Mol. Biol. Evol. 22, 1208-1222. doi: 10.1093/molbev/msil05

Kosakovsky Pond, S. L., and Frost, S. D. W. (2005b). Datamonkey: rapid detection of selective pressure on individual sites of codon alignments. Bioinformatics 21, 2531-2533. doi: 10.1093/bioinformatics/bti320

Kosakovsky Pond, S. L., Frost, S. D. W., and Muse, S. V. (2005). HyPhy: hypothesis testing using phylogenies. Bioinformatics 21, 676-679. doi: 10.1093/bioinformatics/bti079

Liu, H., Xu, Y., Xu, Z., and Chong, K. (2007). A rice YABBY gene, OsYABBY4, preferentially expresses in developing vascular tissue. Dev. Genes Evol. 217, 629-637. doi: 10.1007/s00427-007-0173-0

Lyons, E., and Freeling, M. (2008). How to usefully compare homologous plant genes and chromosomes as DNA sequences. Plant J. 53, 661-673. doi: 10.1111/j.1365-313X.2007.03326.x

Nakayama, H., Yamaguchi, T., and Tsukaya, H. (2010). Expression patterns of $A a D L$, a CRABS CLAW ortholog in Asparagus asparagoides (Asparagaceae), demonstrate a stepwise evolution of CRC/DL subfamily of YABBY genes. Am. J. Bot. 97, 591-600. doi: 10.3732/ajb.0900378

Navarro, C., Efremova, N., Golz, J. F., Rubiera, R., Kuckenberg, M., Castillo, R., et al. (2004). Molecular and genetic interactions between STYLOSA and GRAMINIFOLIA in the control of Antirrhinum vegetative and reproductive development. Development 131, 3649-3659. doi: 10.1242/dev. 01205

Rambaut, A., Suchard, M. A., Xie, D., and Drummond, A. J (2014). Tracer v1.6. Available online at://beast.bio.ed.ac.uk/Tracer

Ronquist, F., and Huelsenbeck, J. P. (2003). MrBayes 3: bayesian phylogenetic inference under mixed models. Bioinformatics 19, 1572-1574. doi: 10.1093/bioinformatics/btg180

Sawa, S., Ito, T., Shimura, Y., and Okada, K. (1999b). FILAMENTOUS FLOWER controls the formation and development of Arabidopsis inflorescences and floral meristems. Plant Cell 11, 69-86. doi: 10.2307/3870839

Sawa, S., Watanabe, K., Goto, K., Liu, Y. G., Shibata, D., Kanaya, E., et al. (1999a). FILAMENTOUS FLOWER, a meristem and organ identity gene of Arabidopsis, encodes a protein with a zinc finger and HMG-related domains. Genes Dev. 13, 1079-1088. doi: 10.1101/gad.13.9.1079 
Siegfried, K. R., Eshed, Y., Baum, S. F., Otsuga, D., Drews, G. N., and Bowman, J. L. (1999). Members of the YABBY gene family specify abaxial cell fate in Arabidopsis. Development 126, 4117-4128.

Specht, C. D., Yockteng, R., Almeida, A. M., Kirchoff, B. K., and Kress, J. W. (2012). Homoplasy, pollination, and emerging complexity during the evolution of floral development in the tropical gingers (Zingiberales). Bot. Rev. 78, 440-462. doi: 10.1007/s12229-012-9111-6

Tononi, P., Möller, M., Bencivenga, S., and Spada, A. (2010). GRAMINIFOLIA homolog expression in Streptocarpus rexii is associated with the basal meristems in phyllomorphs, a morphological novelty in Gesneriaceae. Evol. Dev. 12, 61-73. doi: 10.1111/j.1525-142X.2009.00391.x

Toriba, T., Harada, K., Takamura, A., Nakamura, H., Ichikawa, H., Suzaki, T. et al. (2007). Molecular characterization the YABBY gene family in Oryza sativa and expression analysis of OsYABBY1. Mol. Genet. Genomics 277, 457-468. doi: 10.1007/s00438-006-0202-0

Villanueva, J. C., Broadhvest, J., Hauser, B. A., Meister, R. J., Schneitz, K., and Gasser, C. S. (1999). INNER NO OUTER regulates abaxial-adaxial patterning in Arabidopsis ovules. Genes Dev. 13, 3160-3169. doi: 10.1101/gad.13.23.3160

Waites, R., and Hudson, A. (1995). Phantastica: a gene required for dorsoventrality of leaves in Antirrhinum majus. Development 121, 2143-2154.

Worden, A. Z., Lee, J. H., Mock, T., Rouzé, P., Simmons, M. P., Aerts, A. L. et al. (2009). Green evolution and dynamic adaptations revealed by genomes of the marine picoeukaryotes Micromonas. Science 324, 268-272. doi: $10.1126 /$ science. 1167222

Yamada, T., Ito, M., and Kato, M. (2003). Expression pattern of INNER NO OUTER homologue in Nymphaea (water lily family, Nymphaeaceae). Dev. Genes Evol. 213, 510-513. doi: 10.1007/s00427-003-0350-8

Yamada, T., Ito, M., and Kato, M. (2004). YABBY2-homologue expression in lateral organs of Amborella trichopoda (Amborellaceae). Int. J. Plant Sci. 165, 917-924. doi: $10.1086 / 423793$
Yamada, T., Yokota, S., Hirayama, Y., Imaichi, R., Kato, M., and Gasser, C. S. (2011). Ancestral expression patterns and evolutionary diversification of YABBY genes in angiosperms. Plant J. 67, 26-36. doi: 10.1111/j.1365313X.2011.04570.x

Yamaguchi, T., Nagasawa, N., Kawasaki, S., Matsuoka, M., Nagato, Y., and Hirano, H. Y. (2004). The YABBY gene DROOPING LEAF regulates carpel specification and midrib development in Oryza sativa. Plant Cell 16, 500-509. doi: $10.1105 /$ tpc. 018044

Yamaguchi, T., Nukazuka, A., and Tsukaya, H. (2012). Leaf adaxialabaxial polarity specification and lamina outgrowth: evolution and development. Plant Cell Physiol. 53, 1180-1194. doi: 10.1093/pcp/ pcs074

Yang, Z. (2007). PAML 4: a program package for phylogenetic analaysis by maximum likelihood. Mol. Biol. Evol. 24, 1586-1591. doi: 10.1093/molbev/msm088

Yockteng, R., Almeida, A. M. R., Yee, S., Andre, T., Hill, C., and Specht, C. D. (2013). A method for extracting high-quality RNA from diverse plants for nextgeneration sequencing and gene expression analyses. Appl. Plant Sci. 1:1300070. doi: 10.3732/apps.1300070

Conflict of Interest Statement: The authors declare that the research was conducted in the absence of any commercial or financial relationships that could be construed as a potential conflict of interest.

Copyright $\odot 2015$ Morioka, Yockteng, Almeida and Specht. This is an open-access article distributed under the terms of the Creative Commons Attribution License (CC $B Y)$. The use, distribution or reproduction in other forums is permitted, provided the original author(s) or licensor are credited and that the original publication in this journal is cited, in accordance with accepted academic practice. No use, distribution or reproduction is permitted which does not comply with these terms. 